ANNALES

POLONICI MATHEMATICI

$87(2005)$

\title{
The Jacobian Conjecture: symmetric reduction and solution in the symmetric cubic linear case
}

\author{
by LUDWIK M. DRUŻKOWSKI (Kraków)
}

To the memory of Professor Stanisław Eojasiewicz

\begin{abstract}
Let $\mathbb{K}$ denote $\mathbb{R}$ or $\mathbb{C}, n>1$. The Jacobian Conjecture can be formulated as follows: If $F: \mathbb{K}^{n} \rightarrow \mathbb{K}^{n}$ is a polynomial map with a constant nonzero jacobian, then $F$ is a polynomial automorphism. Although the Jacobian Conjecture is still unsolved even in the case $n=2$, it is convenient to consider the so-called Generalized Jacobian Conjecture (for short (GJC)): the Jacobian Conjecture holds for every $n>1$. We present the reduction of (GJC) to the case of $F$ of degree 3 and of symmetric homogeneous form and prove (JC) for maps having cubic linear form with symmetric $F^{\prime}(x)$, more precisely: polynomial maps of cubic linear form with symmetric $F^{\prime}(x)$ and constant nonzero jacobian are tame automorphisms.
\end{abstract}

1. Formulation and basic facts. Let $\mathbb{K}$ denote either $\mathbb{C}$ or $\mathbb{R}$ and let $F_{j} \in \mathbb{K}\left[X_{1}, \ldots, X_{n}\right], j=1, \ldots, n$. We put $F=\left(F_{1}, \ldots, F_{n}\right): \mathbb{K}^{n} \rightarrow \mathbb{K}^{n}$, $\operatorname{Jac} F(x):=\operatorname{det}\left[\frac{\partial F_{i}}{\partial x_{j}}(x): i, j=1, \ldots, n\right]$ and denote by $\mathcal{P}\left(\mathbb{K}^{n}\right)$ the set of all polynomial transformations of $\mathbb{K}^{n}$. Now we recall the formulation of the $n$-dimensional Jacobian Conjecture, for short $(\mathrm{JC})_{n}$, for $n \geq 2$ :

$$
\left[F \in \mathcal{P}\left(\mathbb{K}^{n}\right) \text {, Jac } F=\text { const } \neq 0\right] \Rightarrow[F \text { is injective }] .
$$

and the so-called Generalized Jacobian Conjecture, for short (GJC):

$(\mathrm{JC})_{n}$ holds for every $n \geq 2$.

Note that the Jacobian Conjecture is on Smale's list of "Mathematical Problems for the Next Century" as Problem 16 among 18 problems ([Sm]). It is known that any injective polynomial map of $\mathbb{K}^{n}$ is bijective ([BR], $[\mathrm{KR}]$ ) and, moreover, each injective polynomial map $F$ of $\mathbb{C}^{n}$ is a polynomial automorphism, i.e. the inverse $F^{-1}$ exists and is a polynomial mapping ([BCW], [E], [W], [Y]). If $F$ is a polynomial automorphism, then $\operatorname{deg} F^{-1} \leq(\operatorname{deg} F)^{n-1}$ ([BCW], [E], [RW]).

2000 Mathematics Subject Classification: 14R15, 14R10.

Key words and phrases: Jacobian Conjecture, cubic linear form, symmetric reduction. 
If $\mathbb{K}=\mathbb{R}$ and $\operatorname{Jac} F(x)>0$ for any $x \in \mathbb{R}^{n}$, then we can ask about injectivity of $F$, which is the so-called Strong Real Jacobian Problem. In 1994, Pinchuk ([Pi]) gave an example showing that the Strong Real Jacobian Problem has a negative answer even in the case of $\mathbb{R}^{2}$, so also in $\mathbb{R}^{n}$.

Using the Lefschetz Principle one can check that the formulation of the Jacobian Conjecture for the field $\mathbb{C}$ covers the case of the Jacobian Conjecture formulated for any field/domain of characteristic zero. Note that to date the Jacobian Conjecture remains unsolved even if $n=2$. For a nice survey containing also other equivalent formulations of the Jacobian Conjecture we refer the reader to $[\mathrm{E}]$.

Since $F \in \mathcal{P}\left(\mathbb{C}^{n}\right)$ can be treated as $\widehat{F}=(\operatorname{Re} F, \operatorname{Im} F) \in \mathcal{P}\left(\mathbb{R}^{2 n}\right)$ and Jac $\widehat{F}(x, y)=|\operatorname{Jac} F(x+i y)|^{2}$, it is evident that

$$
(\mathrm{JC})_{2 n} \text { for } \mathbb{R}\left[X_{1}, \ldots, X_{2 n}\right] \Rightarrow(\mathrm{JC})_{n} \text { for } \mathbb{C}\left[X_{1}, \ldots, X_{n}\right] \text {, }
$$

so the "real (GJC)" implies the "complex (GJC)". But we even do not know if the following ("small (JC)") is true.

Problem 1.1. For any $n>1$, real $(\mathrm{JC})_{n} \Rightarrow \operatorname{complex}(\mathrm{JC})_{n}$.

2. Reduction of the degree. We recall some known reduction theorems that are used in the investigation of (GJC), and prove the theorem on symmetric homogeneous reduction. Note that it is sufficient to consider in (JC) only polynomial mappings of the form $F(x)=x+Q(x)$, where there is no constant or linear term in $Q$ (since instead of $F$ we can investigate $\left.G(x)=\left[F^{\prime}(0)\right]^{-1} \circ[F(x)-F(0)]\right)$. If $F=\left(F_{1}, \ldots, F_{n}\right)$, then we denote by $\operatorname{deg} F$ the number $\max \left\{\operatorname{deg} F_{j}: j=1, \ldots, n\right\}$.

Theorem 2.1 ([BCW], [Y] ). Let $F=\left(F_{1}, \ldots, F_{n}\right): \mathbb{K}^{n} \rightarrow \mathbb{K}^{n}$. Then there exist a natural number $N \geq n$ and polynomial automorphisms $P$ and $Q$ of $\mathbb{K}^{n}$ such that $\operatorname{Jac} A=\operatorname{Jac} B=1$ and $\operatorname{deg} P \circ \widehat{F} \circ Q \leq 3$, where $\widehat{F}=$ $\left(F, I_{N-n}\right): \mathbb{K}^{n} \times \mathbb{K}^{N-n} \ni(x, y) \mapsto(F(x), y) \in \mathbb{K}^{n} \times \mathbb{K}^{N-n}$.

COROLlaRY 2.2. It is enough to check (GJC) for polynomial mappings of degree 3 .

The procedure of adding new variables (as in the above theorem) to obtain a simplified class of objects in a given problem is called the stabilization method. Using this method one can get a further simplification.

TheOrem 2.3 ([Y, BCW, D1]). If we consider the Generalized Jacobian Conjecture, then it is sufficient to consider, for every $n>1$, only polynomial mappings of the so-called cubic homogeneous form $F=I+H$, where $I$ denotes the identity map, $H=\left(H_{1}, \ldots, H_{n}\right)$ and $H_{j}: \mathbb{K}^{n} \rightarrow \mathbb{K}$ is a cubic homogeneous polynomial of degree 3 or $H_{j}=0, j=1, \ldots, n$.

We have the following. 
Proposition 2.4. Let $F=\left(F_{1}, \ldots, F_{n}\right) \in \mathcal{P}\left(\mathbb{K}^{n}\right)$ be a quadratic map, i.e. $\operatorname{deg} F:=\max \left\{\operatorname{deg} F_{j}: j=1, \ldots, n\right\} \leq 2$. Then $F$ is injective if and only if $\operatorname{Jac} F(x) \neq 0$ for any $x \in \mathbb{K}^{n}$.

Proof. This follows immediately from the identity

$$
F(x)-F(y)=F^{\prime}\left(\frac{x+y}{2}\right)(x-y) \quad \text { for every } x, y \in \mathbb{K}^{n} .
$$

As a consequence of the above proposition and Pinchuk's example ([Pi]) mentioned above we get

COROLlaRY 2.5. It is impossible to reduce (by the stabilization method) any polynomial mapping $F \in \mathcal{P}\left(\mathbb{K}^{n}\right)$ to a quadratic map $\widehat{F} \in \mathcal{P}\left(\mathbb{K}^{N}\right)(N \geq n)$ preserving injectivity and nowhere vanishing jacobian.

It is easy to prove the following:

Proposition 2.6 ([BCW, D1]). Let $F=I+H$ have a cubic homogeneous form. Then

$$
\operatorname{Jac} F=1 \Leftrightarrow H^{\prime}(x) \text { is a nilpotent matrix for any } x \in \mathbb{K}^{n} .
$$

Note that $H^{\prime}(x)=3 \widetilde{H}(x, x, \cdot)$, where $\widetilde{H}$ denotes the unique symmetric 3-linear mapping such that $\widetilde{H}(x, x, x)=H(x)$. Hence, if $\operatorname{Jac}(I+H)=1$, then by Proposition 2.6 the matrix

$$
H_{x}:=\widetilde{H}(x, x, \cdot)=\frac{1}{3} H^{\prime}(x) \text { is nilpotent. }
$$

Thus, for every $x \in \mathbb{K}^{n}$, there exists a natural number $p(x)$ such that $H_{x}^{p(x)}$ $=0$ and $H_{x}^{p(x)-1} \neq 0$. It is evident that $1 \leq p(x) \leq 1+\operatorname{rank} H_{x} \leq n$ for every $x \in \mathbb{K}^{n}$. We define the index of nilpotency of the mapping $F=I+H$ to be

$$
\text { ind } F:=\sup \left\{p(x) \in \mathbb{N}: H_{x}^{p(x)}=0, H_{x}^{p(x)-1} \neq 0, x \in \mathbb{K}^{n}\right\} .
$$

If $v=\left(v_{1}, \ldots, v_{n}\right)^{T}$ is a column vector and $k \in \mathbb{N}$, then we denote by $v^{\circ k}$ the $k$ th Hadamard-Schur power of $v$, i.e. $v^{\circ k}:=\left(\left(v_{1}\right)^{k}, \ldots,\left(v_{n}\right)^{k}\right)^{T}$, and by $\Delta\left(v^{\circ k}\right)$ the diagonal $n \times n$ matrix

$$
\Delta\left(v^{\circ k}\right):=\left(\begin{array}{ccccc}
\left(v_{1}\right)^{k} & 0 & \ldots & 0 & 0 \\
0 & \left(v_{2}\right)^{k} & \ldots & 0 & 0 \\
\ldots \ldots \ldots & \ldots & \ldots \ldots \ldots \ldots \ldots \\
0 & 0 & \ldots & \left(v_{n-1}\right)^{k} & 0 \\
0 & 0 & \ldots & 0 & \left(v_{n}\right)^{k}
\end{array}\right) .
$$

Now we present a theorem which allows us to reduce the verification of the Generalized Jacobian Conjecture to the investigation of polynomial mappings of the so-called cubic linear form. 
Theorem 2.7 (cubic linear fomulation of (GJC), [D1-D3]).

(i) In order to verify (GJC) it is sufficient to check it only for polynomial mappings $F=\left(F_{1}, \ldots, F_{n}\right)$ of cubic linear form, i.e.

$(\mathrm{CLF})$

$$
F(x)=\left(\begin{array}{c}
x_{1}+\left(a_{1} x\right)^{3} \\
x_{2}+\left(a_{2} x\right)^{3} \\
\vdots \\
x_{n}+\left(a_{n} x\right)^{3}
\end{array}\right),
$$

where $x \in \mathbb{K}^{n}, a_{j}=\left(a_{j}^{1}, \ldots, a_{j}^{n}\right) \in \mathbb{K}^{n}, a_{j} x:=a_{j}^{1} x_{1}+\cdots+a_{j}^{n} x_{n}$, $j=1, \ldots, n$. Briefly

$$
F(x)=x+(A x)^{\circ 3},
$$

where $A:=\left[a_{i}^{j}: i, j=1, \ldots, n\right], x \in \mathbb{K}^{n}$ and $(A x)^{T}:=\left(a_{1} x, \ldots, a_{n} x\right)$, i.e. Ax is a one-column matrix/vector.

(ii) Without loss of generality we can additionally assume in (i) that $A$ has an additional nilpotency property, namely there exists $c \in \mathbb{K}^{n}$ such that

$$
A=\left.\left((A x)^{\circ 3}\right)^{\prime}\right|_{x=c}=3 \Delta\left((A c)^{\circ 2}\right) A=\left[\begin{array}{ccc}
3\left(a_{1} c\right)^{2} a_{1}^{1} & \ldots & 3\left(a_{1} c\right)^{2} a_{1}^{n} \\
\ldots \ldots \ldots \ldots \ldots & \ldots \ldots \ldots \ldots \ldots \\
3\left(a_{n} c\right)^{2} a_{n}^{1} & \ldots & 3\left(a_{n} c\right)^{2} a_{n}^{n}
\end{array}\right]
$$

and ind $A=$ ind $F$.

(iii) Without loss of generality we can additionally assume in (i) that $A^{2}=0$.

REMARK 2.8. (i) Note that if you take $A$ in (i) such that $A^{2}=0$, then usually $2=$ ind $A<$ ind $F$.

(ii) The cubic homogeneous form (Yagzhev's form) is invariant under the action of the full linear group $\mathrm{GL}_{n}(\mathbb{K})$, i.e. if $F$ has a cubic homogeneous form and $L \in \mathrm{GL}_{n}(\mathbb{K})$, then $L \circ F \circ L^{-1}$ also has a cubic homogeneous form. The cubic linear form is not invariant under the action of $\mathrm{GL}_{n}(\mathbb{K})$, but it is invariant under the action of the subgroup generated by all permutations and dilations $J\left(x_{1}, \ldots, x_{n}\right)=\left(c_{1} x_{1}, \ldots, c_{n} x_{n}\right)$.

Up to now we do not even know whether polynomials $F_{j}(x)=x_{j}+$ $H_{j}(x), j=1, \ldots, n$, are irreducible when $\operatorname{Jac} F=1$ although irreducibility is necessary if $F$ is a polynomial automorphism; the cubic linear form is nicer.

REMARK 2.9. If $F=\left(F_{1}, \ldots, F_{n}\right)$ has (CLF) and Jac $F=1$, then the polynomials $F_{j}(x)=x_{j}+\left(a_{j} x\right)^{3}, j=1, \ldots, n$, are always irreducible. 
The mapping $F$ is a tame automorphism if the mapping $F-F(0)$ is a finite composition of linear automorphisms and shears (also called elementary automorphisms). A shear is an elementary triangular transformation of the form

$T\left(x_{1}, \ldots, x_{n}\right)=\left(x_{1}, \ldots, x_{i-1}, x_{i}+f\left(x_{1}, \ldots, x_{i-1}, x_{i+1}, \ldots, x_{n}\right), x_{i+1}, \ldots, x_{n}\right)$.

Now we recall a theorem which summarizes some partial results on the Generalized Jacobian Conjecture contained in [D1, D2, DR].

TheOREM 2.10. For any $n>1$, if a polynomial map $F=\left(F_{1}, \ldots, F_{n}\right)$ : $\mathbb{K}^{n} \rightarrow \mathbb{K}^{n}$ with Jac $F=1$ has a cubic linear form and if

$$
\operatorname{rank} A<3 \text { or } \quad \operatorname{corank} A<3 \text { or } \quad \text { ind } F=1,2,3, n \text {, }
$$

then $F$ is a tame polynomial automorphism.

Combining Theorem 2.10 with Hubbers' results (cf. [E]) we obtain the following.

Corollary 2.11. If $F(x)=x+(A x)^{\circ 3}: \mathbb{K}^{n} \rightarrow \mathbb{K}^{n}$ and Jac $F=1$, then $F$ is an automorphism provided $n<8$.

3. Symmetric reduction of (GJC). We show that it is possible to reduce (GJC) to complex cubic homogeneous Yagzhev's form with symmetric jacobian matrix $F^{\prime}(x)$ for any $x \in \mathbb{C}^{n}$, even if we start from $F: \mathbb{R}^{n} \rightarrow \mathbb{R}^{n}$.

Let $M_{p, q}(\mathbb{K})$ denote the set of $p$ (rows) $\times q$ (columns) matrices with coefficients in $\mathbb{K}, M_{p}(\mathbb{K}):=M_{p, p}(\mathbb{K})$.

If $Q:=Q^{[0]}+Q^{[1]}+\cdots+Q^{[d]}: \mathbb{K}^{n} \rightarrow \mathbb{K}$ is a nonzero polynomial with homogeneous terms $Q^{[j]}$ of degree $j$, then we put ord $Q:=\min \left\{j: Q^{[j]} \neq 0\right.$, $j=0, \ldots, d\}$.

THEOREM 3.1.

(i) It is sufficient to verify (GJC) only for polynomial mappings of real symmetric cubic homogeneous form, $F(x, y)=(-x, y)+S(x, y)$ : $\mathbb{R}^{2 n} \rightarrow \mathbb{R}^{2 n}$ (for every $n>1$ ), where $(x, y) \in \mathbb{R}^{n} \times \mathbb{R}^{n}=\mathbb{R}^{2 n}$, $S=\left(S_{1}, \ldots, S_{2 n}\right): \mathbb{R}^{2 n} \rightarrow \mathbb{R}^{2 n}$ is a cubic homogeneous polynomial mapping of degree 3 and, for any $(x, y) \in \mathbb{R}^{2 n}, S^{\prime}(x, y)$ is a real symmetric matrix.

(ii) It is sufficient to verify (GJC) only for polynomial mappings of complex symmetric cubic homogeneous form, $F(x)=x+\widehat{S}(x)$ : $\mathbb{C}^{2 n} \rightarrow \mathbb{C}^{2 n}$ (for every $n>1$ ), where $\widehat{S}: \mathbb{C}^{2 n} \rightarrow \mathbb{C}^{2 n}$ is a cubic homogeneous polynomial mapping of degree 3 and, for any $x \in \mathbb{C}^{2 n}$, $\widehat{S}^{\prime}(x)$ is a complex symmetric nilpotent matrix.

Proof. Note that $A=\left[\begin{array}{ll}a & b \\ c & d\end{array}\right] \in M_{p+r, p+r}(\mathbb{K})$ is a symmetric matrix if and only if $a=a^{T} \in M_{p, p}, c=b^{T} \in M_{r, p}, d=d^{T} \in M_{r, r}$. Let $x=\left(x_{1}, \ldots, x_{n}\right)^{T}$, 
$v=\left(v_{1}, \ldots, v_{n}\right)^{T}$ and let $F=\left(F_{1}, \ldots, F_{n}\right)^{T}$ be a polynomial mapping of $\mathbb{K}^{n}$ (the transpose ${ }^{T}$ indicates that we treat $x, v, F$ as a column vector/matrix). It is obvious that without loss of generality we can consider $F$ of the form

$$
F(x)=\left(x_{1}+Q_{1}(x), \ldots, x_{n}+Q_{n}(x)\right)^{T}: \mathbb{K}^{n} \rightarrow \mathbb{K}^{n},
$$

where $Q_{j}$ is a polynomial with ord $Q_{j} \geq 2, j=1, \ldots, n$.

Take

$$
g(v, x):=v_{1} F_{1}(x)+\cdots+v_{n} F_{n}(x)
$$

and define $G \in \mathcal{P}\left(\mathbb{K}^{2 n}\right)$ by the formula

$$
G(v, x):=\nabla g(v, x)^{T}=\left(\frac{\partial g}{\partial v_{1}}, \ldots, \frac{\partial g}{\partial v_{n}}, \frac{\partial g}{\partial x_{1}}, \ldots, \frac{\partial g}{\partial x_{n}}\right)^{T} .
$$

One can easily verify that

$$
\begin{aligned}
G(v, x) & =\left(F_{1}(x), \ldots, F_{n}(x), \sum_{k=1}^{n} v_{k} \frac{\partial F_{k}}{\partial x_{1}}, \ldots, \sum_{k=1}^{n} v_{k} \frac{\partial F_{k}}{\partial x_{n}}\right)^{T} \\
& =\left(F(x),\left[F^{\prime}(x)\right]^{T} v\right) .
\end{aligned}
$$

Obviously, $G$ is injective if and only if $F$ is. We calculate

$$
G^{\prime}(v, x)=\left[\begin{array}{cc}
0 & F^{\prime}(x) \\
F^{\prime}(x)^{T} & {\left[F^{\prime \prime}(x)\right]^{T}(v, \cdot)}
\end{array}\right],
$$

where

$$
\left[F^{\prime \prime}(x)\right]^{T}(v, \cdot)=\left[\begin{array}{ccc}
\sum_{k=1}^{n} v_{k} \frac{\partial^{2} F_{k}}{\partial x_{1}^{2}} & \ldots & \sum_{k=1}^{n} v_{k} \frac{\partial^{2} F_{k}}{\partial x_{n} \partial x_{1}} \\
\ldots \ldots \ldots \ldots \ldots \ldots \ldots \ldots \ldots \ldots \ldots \ldots \ldots \ldots \ldots \ldots \ldots \ldots \\
\sum_{k=1}^{n} v_{k} \frac{\partial^{2} F_{k}}{\partial x_{1} \partial x_{n}} & \ldots & \sum_{k=1}^{n} v_{k} \frac{\partial^{2} F_{k}}{\partial x_{n}^{2}}
\end{array}\right]
$$

is a symmetric $n \times n$ matrix since $\partial^{2} F_{k} / \partial x_{j} \partial x_{i}=\partial^{2} F_{k} / \partial x_{i} \partial x_{j}$. Hence $G^{\prime}(v, x)$ is a symmetric $2 n \times 2 n$ matrix and

$$
G^{\prime}(0,0)=\left[\begin{array}{ll}
0 & I \\
I & 0
\end{array}\right]
$$

where $I$ denotes the $n \times n$ identity matrix. Thus taking the orthogonal matrix

$$
M=\frac{1}{\sqrt{2}}\left[\begin{array}{cc}
I & I \\
-I & I
\end{array}\right]
$$

we get

$$
M^{T} \circ G^{\prime}(0,0) \circ M=E,
$$


where

$$
E=\left[\begin{array}{cc}
-I & 0 \\
0 & I
\end{array}\right]
$$

Put $w=(v, x) \in \mathbb{K}^{2 n}$ and $P(w):=M \circ G \circ M^{T}(w)=E(w)+S(w)$ : $\mathbb{K}^{2 n} \rightarrow \mathbb{K}^{2 n}$. Then the map $P=E+S$ is polynomial and, for any $w \in \mathbb{K}^{2 n}$, $S^{\prime}(w)$ is a symmetric matrix. Note that by the Laplace theorem $\operatorname{Jac} P=$ $\operatorname{Jac} G=(-1)^{n}(\mathrm{Jac} F)^{2}$ and $F$ is injective if and only if $P$ is injective.

(i) Obviously, if $Q: \mathbb{R}^{n} \rightarrow \mathbb{R}^{n}$ is a cubic homogeneous mapping, then $S: \mathbb{R}^{2 n} \rightarrow \mathbb{R}^{2 n}$ is also a cubic homogeneous mapping and, for any $w \in \mathbb{R}^{2 n}$, $S^{\prime}(w)$ is a real symmetric matrix.

(ii) Now it is evident that if we want to get (by similarity) the identity matrix instead of $E$ and to preserve a symmetric form of the matrix $S^{\prime}$ we have to use some complex matrix, e.g. the complex dilation

$$
J=\left[\begin{array}{ll}
i I & 0 \\
0 & I
\end{array}\right]
$$

Put $\widehat{P}(z):=J \circ P \circ J(z)=x+\widehat{S}(z)$, where $\widehat{S}=J \circ S \circ J: \mathbb{C}^{2 n} \rightarrow \mathbb{C}^{2 n}$. Evidently, if $S: \mathbb{R}^{2 n} \rightarrow \mathbb{R}^{2 n}$ is a cubic homogeneous mapping and, for any $w \in \mathbb{R}^{2 n}, S^{\prime}(w)$ is a real symmetric matrix, then $\widehat{S}: \mathbb{C}^{2 n} \rightarrow \mathbb{C}^{2 n}$ is a cubic homogeneous mapping and $\widehat{S}^{\prime}(z)$ is a complex symmetric matrix for any $z \in \mathbb{C}^{2 n}$. Evidently, $F$ is injective if and only if $\widehat{P}$ is injective. Since Jac $\widehat{P}=i^{2 n}(-1)^{n}(\operatorname{Jac} F)^{2}=(\operatorname{Jac} F)^{2}=1$, for any $z \in \mathbb{C}^{2 n}$ the matrix $\widehat{S}^{\prime}(z)$ is a complex symmetric matrix which is also nilpotent.

REMARK 3.2. The trick with a polynomial $g(v, x)$ (which is probably a folklore result) comes from $[\mathrm{M}]$ where the sketch of the reduction of $(\mathrm{JC})_{n}$ to the complex symmetric case of $(\mathrm{JC})_{2 n}$ is given. The reduction of (GJC) to the complex symmetric case has also been proved in [BE].

REMARK 3.3. Let $\left\{e_{1}, \ldots, e_{n}\right\}$ be the canonical basis of the vector space $\mathbb{K}^{n}$. Consider $F$ of cubic homogeneous form, $F(x)=x+H(x), x \in \mathbb{K}^{n}$, and take a unique 3 -linear symmetric mapping $\widetilde{H}=\left(\widetilde{H}_{1}, \ldots, \widetilde{H}_{n}\right): \mathbb{K}^{n} \times$ $\mathbb{K}^{n} \times \mathbb{K}^{n} \rightarrow \mathbb{K}^{n}$ such that $\widetilde{H}(x, x, x)=H(x)$ for any $x \in \mathbb{K}^{n}$. Note that the $\operatorname{map}\left[F^{\prime}(x)\right]^{T} v$ in the definition of the mapping $G$ is equal to

$$
\left[F^{\prime}(x)\right]^{T} v=\left(v_{1}+3 \sum_{j=1}^{n} v_{j} \widetilde{H}_{j}\left(x, x, e_{1}\right), \ldots, v_{n}+3 \sum_{j=1}^{n} v_{j} \widetilde{H}_{j}\left(x, x, e_{n}\right)\right) .
$$

Hence the matrix of the differential of $\left[F^{\prime \prime}(x)\right]^{T}(v, \cdot)$ with respect to $x$ is of the form 


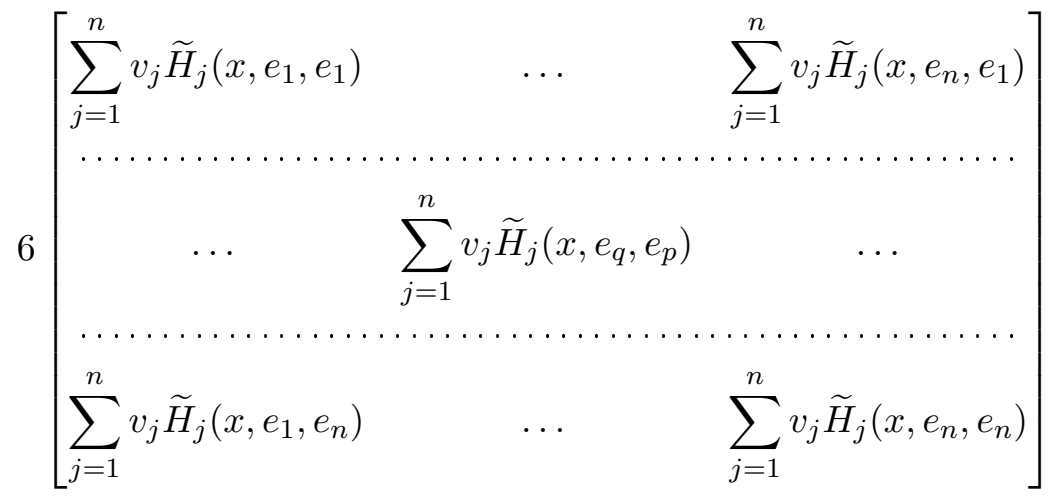

and it is symmetric since $\widetilde{H}(x, u, t)=\widetilde{H}(x, t, u)$.

We know by Theorem 2.7 and Proposition 2.6 that it is sufficient to verify (GJC) for polynomial mappings of the cubic linear form $F(x)=x+(A x)^{\circ} 3$ with nilpotent matrix $\left[(A x)^{\circ 3}\right]^{\prime}=3 \Delta\left((A x)^{\circ 2}\right) A$ for any $x \in \mathbb{K}^{n}$. Although at present we cannot reduce every polynomial mapping to the cubic linear form $F(x)=x+(A x)^{\circ 3}$ with a complex symmetric nilpotent matrix $\left[(A x)^{\circ}\right]^{\prime}$ it is natural, in view of Theorem 3.1, to ask if the Jacobian Conjecture is true in this particular case. We will prove the following.

THEOREM 3.4. If $F(x)=x+(A x)^{\circ 3}: \mathbb{C}^{n} \rightarrow \mathbb{C}^{n}$ is of cubic linear form and the differential $\left[(A x)^{\circ}\right]^{\prime}$ is a complex symmetric nilpotent matrix for any $x \in \mathbb{C}^{n}$, then $F$ is a tame automorphism.

(Note that by Proposition 2.6 the nilpotency of $[F(x)-x]^{\prime}=\left[(A x)^{\circ} 3\right]^{\prime}$ is necessary in our theorem.)

Proof. (0) Notice that if $P$ is a permutation (i.e. the matrix of a permutation) and $F$ has cubic linear form, then the mapping $P^{-1} \circ F \circ P$ also has (CLF). Observe that symmetry and nilpotency of $\left[(A x)^{\circ}\right]^{\prime}$ is preserved since $P^{-1}=P^{T}$ and hence the matrix $\left[P^{T} \circ(A(P x))^{\circ 3}\right]^{\prime}=P^{T} \circ\left[(A y)^{\circ}\right]^{\prime} \circ P$, where $y=P(x)$, is symmetric and nilpotent. Therefore, permutation similarity $P^{-1} \circ F \circ P$ of the mapping $F$ (which is equivalent to taking $P^{-1} \circ A \circ P$ instead of $A$ ) preserves the cubic linear form of $F$ as well as symmetry and nilpotency of the "new" matrix $A$.

Induction. One can check the theorem by simple calculations if $n=2$.

I. If the $i$ th row/column of $A$ is equal to 0 , then-by symmetry-so is the $i$ th column/row of $A$. Due to (0) we can assume that it is the last column and then conclude that $F$ is injective if and only if the mapping $G\left(x_{1}, \ldots, x_{n-1}\right):=\left(F_{1}\left(x_{1}, \ldots, x_{n-1}, 0\right), \ldots, F_{n-1}\left(x_{1}, \ldots, x_{n-1}, 0\right)\right): \mathbb{C}^{n-1}$ $\rightarrow \mathbb{C}^{n-1}$ with symmetric differential is injective. Obviously, $F=\left(G, x_{n}\right) \circ R$, where $R(x)=\left(x_{1}, \ldots, x_{n-1}, x_{n}+F_{n}\left(x_{1}, \ldots, x_{n-1}\right)\right.$. By induction $G$ is a tame automorphism and so is $F$. 
II. Assume that no column/row of $A:=\left[a_{i}^{j}: i, j=1, \ldots, n\right]$ is equal to 0 .

(i) Put $N_{i}=\#\left\{j: j \in\{1, \ldots, i-1, i+1, \ldots, n\}, a_{i}^{j} \neq 0\right\}$ and $N:=$ $\max \left\{N_{i}: i=1, \ldots, n\right\}, q:=\min \left\{i: N=N_{i}\right\}$. If $q=1$ we do nothing. If $q>1$ we exchange the $q$ th row and the first row and then permute the $q$ th column with the first column.

(ii) Now in the first row of the "new" matrix $A$ we have exactly $N$ coefficients different from 0 (we do not count $a_{1}^{1}$ ), say $a_{1}^{j_{1}} \cdots a_{1}^{j_{N}} \neq 0$ where $j_{1}>1$. Permute columns $j_{1}, \ldots, j_{N}$ into columns $2,3, \ldots, N+1$ and the rows in the same way.

(iii) If all columns $a^{N+2}, \ldots, a^{n}$ in the submatrix $B:=\left[a_{i}^{j}: i=1, \ldots\right.$, $N+1, j=1, \ldots, n]$ are equal to 0 , then also by symmetry $a_{i}^{j}=0$ when $i=N+2, \ldots, n, j=1, \ldots, N+1$ and we can represent $F$ as the composition of two mappings

$$
Q(x)=\left(F_{1}\left(x_{1}, \ldots, x_{N+1}\right), \ldots, F_{N+1}\left(x_{1}, \ldots, x_{N+1}\right), x_{N+2}, \ldots, x_{n}\right)
$$

and

$$
R(x)=\left(x_{1}, \ldots, x_{N+1}, F_{N+2}\left(x_{N+2}, \ldots, x_{n}\right), \ldots, F_{n}\left(x_{N+2}, \ldots, x_{n}\right)\right),
$$

i.e. $F=Q \circ R$. By induction $Q$ and $R$ are tame automorphisms, and so is $F$.

(iv) If $s$ of the columns $a^{N+2}, \ldots, a^{n}$ in the submatrix $B$ are not equal to $0,0<s \leq n-N-1$, then we permute them in such a way that only $k$ last columns in $B$ are $0, k:=n-N-1-s \geq 0$. Afterwards we permute the appropriate rows and the "new" matrix $\left[(A x)^{\circ 3}\right]^{\prime}$ is symmetric and nilpotent for any $x \in \mathbb{C}^{n}$.

(v) If $k>0$, then the argument analogous to that given in (iii) finishes the proof.

(vi) If $k=0$, i.e. all columns $a^{N+2}, \ldots, a^{n}$ in $B$ are different from 0 , then it is easy to check that all rows of the symmetric matrix $\left[(A x)^{\circ 3}\right]^{\prime}=$ $3 \Delta\left((A x)^{\circ 2}\right) A$ are different from 0 and parallel to the row $\left(a_{1} x\right)^{2} a_{1}$. Hence $\operatorname{rank} A=1$ and by Theorem 2.10 the mapping $F$ is a tame automorphism.

We formulate another natural question in this direction.

Problem 3.5. Let $F(x)=x+(A x)^{\circ 3}: \mathbb{C}^{n} \rightarrow \mathbb{C}^{n}$ be of cubic linear form, Jac $F=1$ and let the matrix $A$ be a complex symmetric nilpotent matrix. Is then $F$ a tame automorphism?

\section{References}

[BCW] H. Bass, E. H. Connell and D. Wright, The Jacobian Conjecture: reduction of degree and formal expansion of the inverse, Bull. Amer. Math. Soc. 7 (1982), $287-330$. 
[BR] A. Białynicki-Birula and M. Rosenlicht, Injective morphisms of real algebraic varieties, Proc. Amer. Math. Soc. 13 (1962), 200-203.

[BE] M. de Bondt and A. van den Essen, A reduction to the symmetric case, Report 0308 (2003), University of Nijmegen (to appear in Proc. Amer. Math. Soc.).

[D1] L. M. Drużkowski, An effective approach to Keller's Jacobian Conjecture, Math. Ann. 264 (1983), 303-313.

[D2] - , The Jacobian Conjecture in case of rank or corank less than three, J. Pure Appl. Algebra 85 (1993), 233-244.

[D3] - New reduction in the Jacobian Conjecture, Univ. Iagell. Acta Math. 39 (2001), 203-206.

[DR] L. M. Drużkowski and K. Rusek, The formal inverse and the Jacobian Conjecture, Ann. Polon. Math. 46 (1985), 85-90.

[E] A. van den Essen, Polynomial Automorphisms and the Jacobian Conjecture, Birkhäuser, Basel, 2000.

[KR] K. Kurdyka and K. Rusek, Surjectivity of certain injective semialgebraic transformations of $\mathbb{R}^{n}$, Math. Z. 200 (1988), 141-148.

[M] G. Meng, Legendre transform, Hessian conjecture and tree formula, arXiv:mathph/0308035, 28 Aug 2003.

[Pi] S. Pinchuk, A counterexample to the real Jacobian Conjecture, Math. Z. 217 (1994), 1-4.

[RW] K. Rusek and T. Winiarski, Polynomial automorphisms of $\mathbb{C}^{n}$, Univ. Iagell. Acta Math. 24 (1984), 143-149.

[Sm] S. Smale, Mathematical Problems for the Next Century, Math. Intelligencer 20 (1998), 7-15.

[W] T. Winiarski, Inverse of polynomial automorphisms of $\mathbb{C}^{n}$, Bull. Acad. Polon. Sci. Sér. Sci. Math. 27 (1979), 673-674.

[Y] A. V. Yagzhev, On Keller's problem, Sibirsk. Mat. Zh. 21 (1980), 141-150 (in Russian).

Institute of Mathematics

Jagiellonian University

Reymonta 4

30-059 Kraków, Poland

E-mail: Ludwik.M.Druzkowski@im.uj.edu.pl 